KN-9

\section{Complex structures of minerals at Deep Earth conditions: experiments and perspectives}

\author{
Marco Merlini ${ }^{1}$
}

1. Department of Earth Sciences, University of Milano, Milano, Italy email: marco.merlini@unimi.it

The development in the last decades of high-pressure/ high-temperature devices for synthesis and in-situ analysis, allowed the determination of complex structures of minerals stabilized at deep Earth conditions. Furthermore, the discovery in these experiments of unpredicted stoichiometry in Earth's mantle minerals indicate the possible existence of a number of crystalline phases in the planetary interiors unconceivable before. Multiphase and single-phase experiments, combined with conventional and advanced analytical approaches (i.e. X- ray laboratory, X-ray synchrotron and electron single crystal diffraction) are the basis for building an improved mineralogical model for the Earth's interior. Recent results are provided. The first example shows the possible complexity of hydrous mineral phases in subduction environment, where crustal minerals are transferred in the deep Earth, with implication for water storage and transfer in the Earth's interior and consequent large-scale geological processes. The second example about carbonate structures at extreme conditions indicates a complex crystal chemistry with important geochemical implications in the lower mantle. The third example related to iron-oxides reveal a number of unknown stoichiometry in the planetary interiors, with consequence in the global oxidation states of planets. Most of these structures have no structural analogue in natural and synthetic crystalline materials and can stimulate the design of new functional materials.

Keywords: high-pressure, crystal structure, Earth and planetary interior
$\mathrm{KN}-10$

\section{Hydrides: from Hydrogen Storage to Porous Systems, Structural Design and Solid Electrolytes}

Yaroslav Filinchuk ${ }^{1}$

1. Universite catholique de Louvain, Louvain-la-Neuve, Belgium email: yaroslav.filinchuk@uclouvain.be

Hydrogen is traditionally stored in metal hydrides, nowadays seen as too heavy for mobile applications. Complex (ionic solids) and chemical (molecular compounds) hydrides based on light elements, such as $\mathrm{N}$ and $\mathrm{B}$, are developed in the recent years, just like porous solids for physisorption of molecular hydrogen. These compounds gave a way to new applications, which I will highlight "in the light of X-rays", as structural studies are often a clue to understanding the functionality.

Firstly, I will show recent attempts to design a molecular system capable to store hydrogen reversibly. Aluminium is the cheap, light and abundant metal used to store hydrogen in a form of alane $\mathrm{AlH}_{3}$ and alanates $\mathrm{AlH}_{4}^{-}$. We have developed systems based on Al with complex and chemical hydrides coordinated to it, such as ammonia borane $\mathrm{NH}_{3} \mathrm{BH}_{3}$ (AB), amidoborane $\mathrm{NH}_{2} \mathrm{BH}_{3}^{-}$and borohydrides $\mathrm{BH}_{4}^{-}$[1]. We showed [2] that the ability of the strong Lewis acid $\mathrm{Al}^{3+}$ to coordinate both the initial hydrogenated species as well as their dehydrogenation products makes it a good template for chemical transformations of hydrides. In particular, $\mathrm{AB}$ coordinated to $\mathrm{Al}^{3+}$ in $\mathrm{Al}\left(\mathrm{BH}_{4}\right)_{3} \cdot \mathrm{NH}_{3} \mathrm{BH}_{3}$ endothermically dehydrogenates to a single product identified as $\mathrm{Al}\left(\mathrm{BH}_{4}\right)_{3} \cdot \mathrm{N}-$ $\mathrm{HBH}$, with a potential for a direct rehydrogenation of $\mathrm{AB}$.

Secondly, porous materials can be also made of hydrides [3]. We investigated gas sorption in the porous $\gamma-\mathrm{Mg}\left(\mathrm{BH}_{4}\right)_{2}$ using neutron powder diffraction to accurately localize the guests and synchrotron X-ray powder diffraction to collect data along the adsorption isobars. The latter allows to study structural changes with pressure and temperature variation, giving insight into guest-host and guest-guest interactions, as well as to extract relevant thermodynamic parameters. I will discuss the intermolecular interactions, size effects and the role of hydridic hydrogen in physisorption. In this small pore system, the effect of the probe size on the loading capacity and the location of the guest molecules is remarkable. While typically each pore can be occupied by one or two larger guests, the amount of hydrogen can go up to 5 molecules per pore, yielding the total capacity of $2.33 \mathrm{H}_{2}$ molecules per $\mathrm{Mg}$ atom. We are trying to extend the family of hydridic porous solids by combining imidazolates (ligands known to build stable frameworks) and complex hydrides (should be responsible for the unusual functionality). The approach and recent results will be presented.

Thirdly, complex hydrides are known as good solid state electrolytes that may eventually allow to build safe all-solid-state batteries.

References:

[1] Dovgaliuk, I. et al. (2017). ChemSusChem 10, 4725-4734.

[2] Dovgaliuk, I. \& Filinchuk, Y. (2016). Int. J. Hydr. Energy 41, 15489-15504.

[3] Filinchuk, Y. et al. (2011). Angew. Chem. Int. Ed. 50, 1116211166 .

Keywords: hydrides, materials chemistry, design 